\title{
Restoring the harmony of function and aesthetics in partially edentulous arches: A case report
}

\author{
Nikhila KV ${ }^{1 *}$, Rajeswari $\mathrm{CL}^{2}$, Srivatsa $\mathrm{G}^{3}$, Rohit M Shetty ${ }^{4}$ \\ ${ }^{1}$ Post Graduate Student, ${ }^{2}$ Professor, ${ }^{3}$ Professor and Principal, ${ }^{4}$ Professor and HOD, ${ }^{1-4}$ Dept of Prosthodontics, KLE Society's \\ Institute of Dental Sciences, Bangalore, Karnataka, India \\ *Corresponding Author: Nikhila KV \\ Email: nikilak1993@gmail.com
}

\begin{abstract}
Successful prosthetic treatment depends on the functional and esthetic outcomes of the treatment and occlusal plane is a factor that can influence both these outcomes. Identifying and correcting occlusal plane alterations using Broadrick's occlusal plane analyzer positively influences the treatment outcomes. Incorporating aesthetic acetal resin clasp in cast partial dentures is beneficial for patients seeking efficient, affordable and aesthetic treatment option. This article presents a case report where a simplified custom made Broadrick's occlusal analyzer was used to identify the altered occlusal plane and aesthetic acetal resin clasp were given in the maxillary cast partial denture for improved aesthetics.
\end{abstract}

Keywords: Occlusal plane analysis, Fixed partial denture, Cast partial denture, Aesthetic clasp.

\section{Introduction}

Prosthetic rehabilitation of long span edentulous cases requires consideration of various factors for the success of the treatment. One such factor that can affect the functional as well as esthetic outcome of the rehabilitation is the occlusal plane. Delay in replacement of missing teeth can lead to slow alteration in the occlusal plane due to supra-eruption, migration, tipping or rotations. When restorations are added to an existing tooth arrangement characterized by these changes, excursive interferences may be incorporated, resulting in detrimental sequelae. ${ }^{1}$ The curve of Spee, which exists in the ideal natural dentition, allows harmony to exist between the anterior tooth and condylar guidance. ${ }^{2}$ Broadricks Occlusal Plane Analyser provides a simple and practical method to assist in determining the preliminary occlusal plane on diagnostic casts ${ }^{1}$ and assist in the reproduction of tooth morphology that is commensurate with the curve of Spee when posterior restorations are designed. ${ }^{2}$

In partially edentulous patients, although cast removable partial dentures are an effective and affordable treatment option, achieving aesthetics is another challenge that is encountered. Most patients do not accept the metal display of the cast clasps. Acetal (Bio Dentaplast, Bredent, Senden, Germany), a thermoplastic resin, is an alternative denture clasp material that has superior aesthetics that allows clasps to better match the color of abutment tooth. ${ }^{3}$

This case reports presents a partially edentulous case with an altered occlusal plane managed with a simplified custom made Broadrick's occlusal plane analyzer and a subsequent treatment with combined fixed partial dentures and cast partial denture with aesthetic acetal resin clasps.

\section{Case Report}

A 46-year-old female patient reported to the Department of Prosthodontics with a chief complaint of missing teeth and inability to chew since the last 2 years. On clinical examination, it was found that the teeth present were $11,12,18,21,22,23,28$ in the maxillary arch while the mandibular arch had all the teeth except 36,44,45 and 46. (Fig. 1)

Diagnostic cast revealed missing maxillary and mandibular teeth, supra-erupted and tipped mandibular posterior teeth resulting in a deranged occlusal plane.

The following treatment plan was presented to the patient:

1. To perform occlusal plane analysis followed by fabrication of treatment partial denture in maxillary arch 
2. Mandibular- Fixed partial denture w.r.t $35,36,37$ and $44,45,46$ and 47

3. Maxillary - Removable cast partial denture with aesthetic clasp w.r.t 12 and 23

Once the patient approved the treatment plan, the following procedures were done:

An occlusal plane analysis was performed using a custom made Broadrick's occlusal plane analyzer. Custom Broadrick's flag was fabricated using commonly available materials. The orbital pointer was removed and was replaced with a metal rod and stabilized with impression compound. A thick cardboard sheet adhered with white paper was used as flag and was stabilized to the rod with paper clips and an attachment block made with impression compound on the upper member of the articulator. (Fig. 2)

The maxillary cast was removed from the articulator and custom-made flag was attached on top of the upper member of articulator. The anterior survey point (ASP) was chosen on the midpoint of the disto-incisal edge of the mandibular right and left canine from which a long arc of radius 4-inch was drawn on the flag with a compass. The posterior survey point (PSP) was located on the anterior border of condylar element on the articulator and a short arc was drawn from the posterior survey point on the flag to intersect the long arc of anterior survey point. The needle of compass was placed on the point of intersection of both the arc and a four-inch radius line was drawn on the buccal surfaces of right and left mandibular teeth. (Fig. 2)

On the mandibular cast the teeth were reduced to the level of the markings obtained after occlusal analysis maintaining the gross anatomy. Putty index was made for both the right and left sides after adding pontics. An acrylic removable partial denture (RPD) was fabricated on the upper cast mounted against this prepared mandibular cast (Fig. 3). The acrylic RPD was placed in the patients mouth and the mandibular teeth were reduced on both right and left sides until the optimum occlusion as planned on the articulator was obtained (Fig. 4). The mandibular right posterior teeth (44 and 47) were prepared for receiving fixed partial dentures with the opposing acrylic RPD (Fig. 5). The teeth were temporized using the putty index. The fixed partial denture was cemented with the acrylic RPD in place. Similar procedure was followed for left side where 35 and 37 were prepared to receive fixed partial denture.

After the fixed partial dentures were cemented using glass ionomer cement in the mandibular arch, rest seats were prepared on the maxillary teeth w.r.t $12,18,23$ and 28 to receive cast partial denture with aesthetic clasp w.r.t 12 and 23. Try-in of the metal framework was performed (Fig. 6) followed by try-in of the waxed-up trial on metal framework. The partial denture was processed and delivered after doing necessary corrections (Fig. 7). Instructions were given.

24hrs recall followed by 3 days, 7 days, 1 month and 3 months revealed minor pressure spots w.r.t 12 and 23 in the cast partial denture that were relieved.

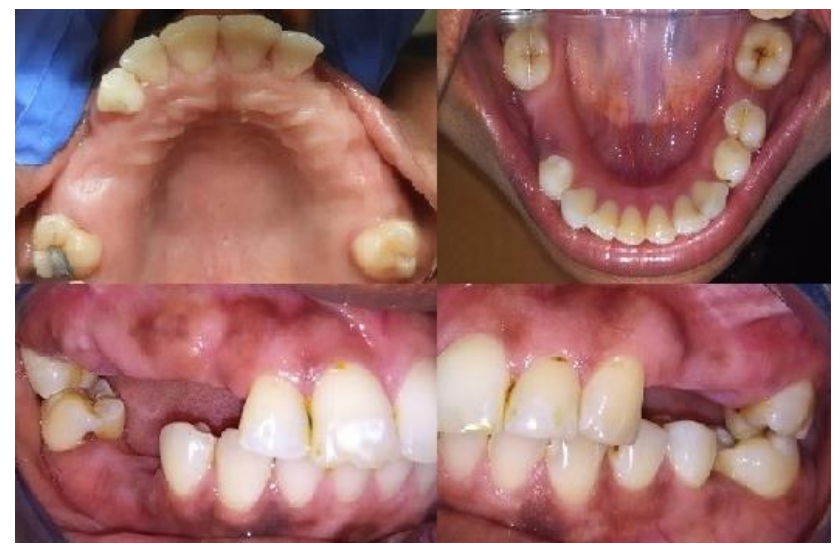

Fig. 1: Represents the Pre-operative intraoral view of the case

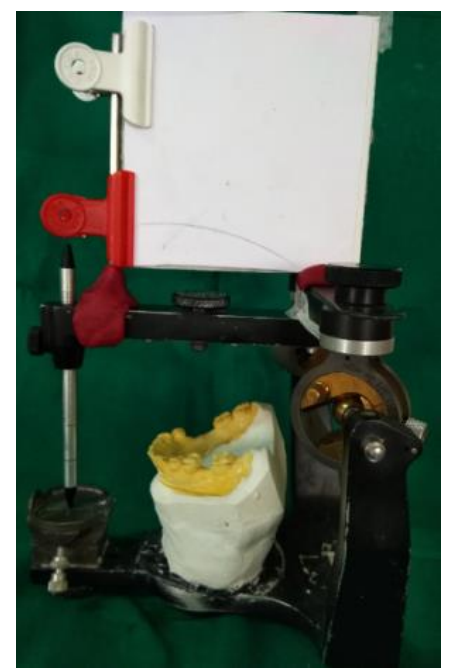

Fig. 2: Custom made Broadrick's flag and Occlusal plane analysis conducted using the custom analyser 


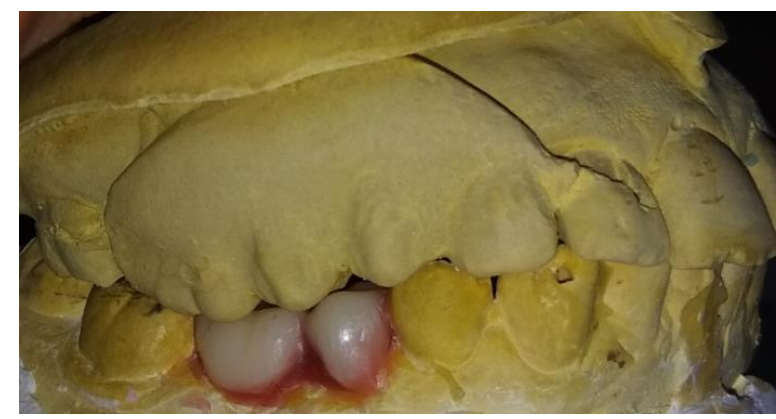

Fig. 3: Pontic added for putty index in the modified mandibular cast

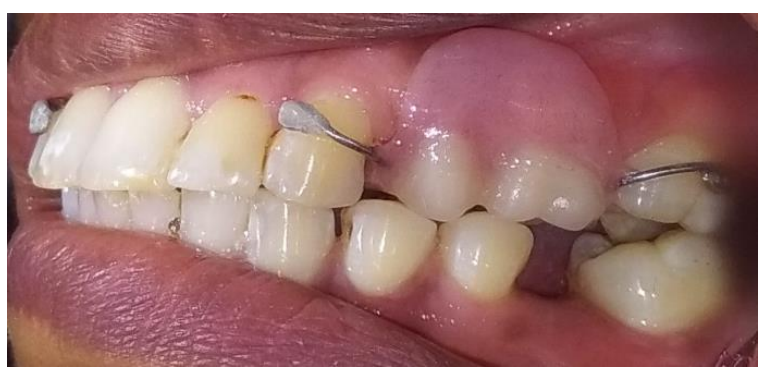

Fig. 4: Acrylic RPD used for reducing the mandibular teeth to correct occlusal plane,

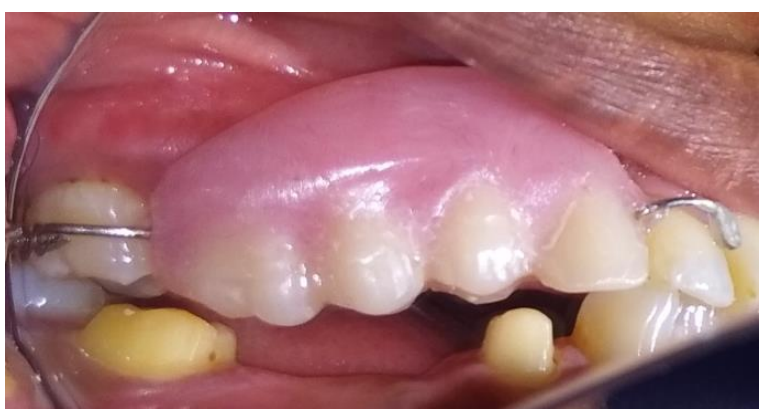

Fig. 5: Acrylic RPD used as guide for occlusal clearance of FPD

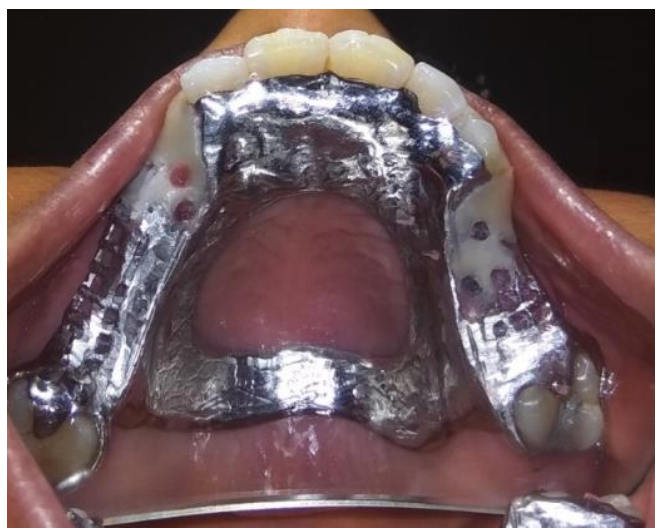

Fig. 6: Framework trial with acetal resin clasp in the upper arch

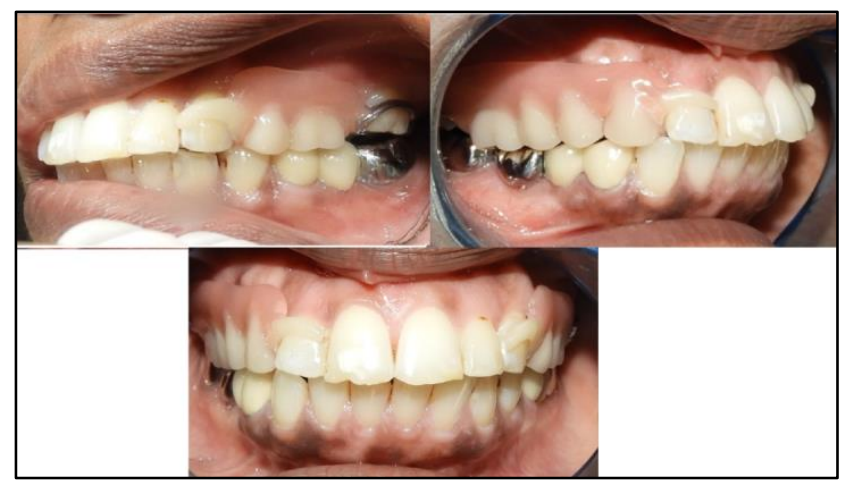

Fig. 7: Final prosthesis with cast removable partial denture in the maxilla and fixed partial denture in the mandibular

\section{Discussion}

Proper management of the occlusal plane is an essential consideration when multiple long-span posterior restorations are designed. ${ }^{5}$ The curve of Spee may be pathologically altered in situations resulting from rotation, tipping, and extrusion of teeth. Restoration of the dentition to such an altered occlusal plane can compromise the prosthesis functionally and aesthetically. The Broadrick Occlusal Plane Analyzer aka Broadrick flag permits reconstruction of the curve of Spee in harmony with the anterior and condylar guidance. ${ }^{1}$ It assists in locating the cusp tips of the posterior teeth. In addition, it can demonstrate how much tooth reduction or porcelain addition is needed to idealize the occlusal plane. ${ }^{1}$ In the present case the clinical and diagnostic cast examination showed alteration of curve of Spee due to supra-eruption and tipping of mandibular teeth into the edentulous space and hence it was decided to do an occlusal analysis with BOPA. The original BOPA (Broadricks flag, Teledyne Water Pik, Fort Collins, Colo) is an expensive instrument and adapted to only a few articulator systems. ${ }^{4,6}$ Therefore, a custom occlusal plane analyser was fabricated by further simplifying the technique given by Chaturvedi et $\mathrm{al}^{4}$ by using commonly available materials like impression compound and cardboard. This simple occlusal analyser helped in assessing and correcting the occlusal plane in the current case.

The occlusal plane correction did not necessitate the need for intentional root canal treatment or crown lengthening for the mandibular teeth. Fixed partial 
dentures were planned for the mandibular posterior missing teeth. The acrylic RPD that was given in the upper arch was a simple method to guide the tooth reduction to correct the occlusal plane and also helped in guiding the amount of clearance required for the fixed partial dentures.

In the maxillary arch, fixed partial denture was not indicated as the periodontal membrane area of the abutment teeth was much lesser than area of missing teeth, violating the Ante's law $^{7}$ and implants were ruled out due to economic reasons. Hence in this case cast removable partial was considered the most effective and affordable treatment in the upper arch. Various studies have stated that patients fail to wear partial dentures as they find the display of the clasps which act as direct retainers esthetically unacceptable. ${ }^{8}$ A similar concern was raised by the patient which led to the decision to use aesthetic alternative for clasp assemblies. Technopolymer clasps manufactured from thermoplastic acetal resin (POM) help in overcoming the esthetics concerns of removable partial denture. Esthetic constitutes as a major advantage of this clasp along with high transverse strength, flexibility and radiolucency. They can be used in larger and deeper undercuts because of low modulus of elasticity and in situations where esthetics and periodontal health are priorities. ${ }^{8}$

\section{Conclusion}

A comprehensive and meticulous diagnosis and treatment planning is very crucial in the success of any prosthetic treatment. The occlusal plane analyzers should be used prior to designing multiple long span posterior restorations for better functional and esthetic outcomes. Each patient's esthetic demands should be considered during the treatment planning so that best alternatives can be decided.

\section{Source of Funding}

None.

\section{Conflict of Interest}

None.

\section{References}

1. Renu Gupta, RP Luthra and Hardik Hitesh Sheth. Broadrick's occlusal plane analyzer: A review. Int J Appl Dent Sci 2019;5(1):95-8.

2. Christopher D. Lynch and Robert J. McConnell. Prosthodontic management of the curve of Spee: Use of the Broadrick flag.

3. K. Lekha, N.P Savitha, Meshramkar Roseline and Ramesh K. Nadiger. Acetal resin as an esthetic clasp material. $J$ Interdiscip Dent 2012;2(1).

4. Chaturvedi S, AK Verma, Ali M, Shah M. Full mouth rehabilitation broadrick flag: A case report. Int J Case Rep Images 2012;5(3).

5. Manvi S, Miglani S, Rajeswari C.L, Srivatsa G, and Arora S. Occlusal Plane Determination Using Custom Made Broadrick Occlusal Plane Analyser: A Case Control Study. ISRN Dentistry 2012

6. R.W Toothaker, DDS, A and Angela R. Graves. Custom adaptation of an occlusal plane analyzer to a semiadjustable articulator. J Prosthet Dent 1999;81:240-2.

7. Herbert T Shillingburg. Fundamentals of fixed prosthodontics. Fourth edition. Quintessence Publishing Co.

8. Turagam N, Mudrakola DP, Yelamanchi RS, Deepthi M, Natarajan M. Esthetic clasp cast partial denture. J Int Soc Prevent Communit Dent 2019; 9(1):94-98.

How to cite this article: Nikhila KV, Rajeswari CL, Srivatsa G, Shetty RM. Restoring the harmony of function and aesthetics in partially edentulous arches: A case report. Ann Prosthodont Restor Dent 2019;5(4):135-8 